\title{
PET myocardial blood flow is now officially declared ready for prime time (and a little bit about how the US coding/valuation/coverage system works)
}

\author{
Randall C. Thompson, MD, FASNC, ${ }^{\mathrm{a}}$ and Friederike K. Keating, $\mathrm{MD}^{\mathrm{b}}$ \\ a St. Luke's Mid America Heart Institute and the University of Missouri - Kansas City, Kansas City, \\ MO \\ b Division of Cardiology, University of Vermont Medical Center, Burlington, VT
}

Received Jun 25, 2018; accepted Jun 26, 2018

doi: $10.1007 / \mathrm{s} 12350-018-1363-\mathrm{z}$

On May 17, 2018, the American Medical Association's Current Procedural Terminology (CPT) Editorial Panel voted to establish a category one CPT add-on code for PET absolute quantitation of myocardial blood flow (AQMBF). ${ }^{1}$ The new code becomes effective from January 1, 2020 and in the interim, PET AQMBF can be reported using the temporary/technical/tracking code, $+0483 \mathrm{~T}$. The successful code change application was led by ASNC and four other medical societies as part of the societies' advocacy efforts on behalf of our patients and our members. For a medical service to achieve CPT category one status, the hurdles are quite high. ${ }^{2}$ The procedure must be performed widely in the United States and there are rigorous requirements for supporting medical literature. Although some work remains to be done for standardization of PET blood flow measurement and reporting, this milestone indicates that AQMBF is considered mainstream and established enough to soon warrant routine billing and routine reimbursement.

\section{WHAT HAPPENS NEXT?}

When the CPT editorial panel establishes a new category one code, the approved code change application is referred to the AMA's Relative Value Scale Update Committee (RUC). ${ }^{3}$ Physicians who perform the new service are surveyed regarding the time and

Reprint requests: Randall C. Thompson, MD, FASNC, St. Luke's Mid America Heart Institute and the University of Missouri Kansas City, 4300 Wornall Rd, Kansas City, MO 64111; rthompson@saintlukes.org

J Nucl Cardiol 2019;26:316-7.

$1071-3581 / \$ 34.00$

Copyright (C) 2018 American Society of Nuclear Cardiology. intensity and the relative effort of work involved in it. The RUC reviews these surveys and makes recommendations regarding valuation for the service to the centers for Medicare and Medicaid services (CMS). CMS usually, but not always, accepts the valuation and major commercial health insurance companies usually follow suit. On occasion, CMS will establish a different value from the one recommended by the RUC, will decide not to cover a service, or will establish their own billing code (a so-called G code). However, in the vast majority of cases, the establishment of a category one CPT code leads to the service being routinely covered by CMS and other health insurance payers.

Broad participation in the process, most commonly through organized medical societies, is needed from practicing physicians, content experts, and those familiar with coding, valuation, and reimbursement processes. This support ensures expert clinical input is acquired to accurately identify the resource costs involved in providing these critical services to our patients. For example, without substantial numbers of physicians responding to the RUC survey, the RUC may be unable to make a recommendation to CMS, which can lead to local CMS carriers and individual insurance companies deciding on their own what reimbursement to offer, or decide not to cover the service at all.

As mentioned above, AWMBF can be reported using a temporary/tracking CPT code until January 2020 (CPT code +0483T). While " $\mathrm{T}$ " codes such as this are not routinely covered by health insurance companies, they can be. Physicians who are interested in billing the service will frequently meet with or otherwise appeal to the carrier advisory committee of their local health insurance companies and ask for coverage. This appeal usually involves offering comparable services which 
already have established fee schedules. ASNC is actively working with individual physicians and local carriers for broader coverage for CPT code $+0483 \mathrm{~T}$.

\section{ARE OTHER PET CPT CODES CHANGING}

The CPT code change application that was submitted by ASNC and other medical societies involved not only AQMBF, but also changes to the code descriptors for PET myocardial metabolism and for PET myocardial perfusion. ${ }^{1}$ An update of these codes was requested by the RUC when they came up for a routine revaluation. In brief, myocardial metabolism (code 78459) and perfusion (codes 78491 and 78492) were updated to reflect current practices and instrumentation. Codes were introduced by the medical societies for good stewardship, as routine updates to keep up with current practice, and to normalize the coding and reimbursement for PET for new indications. The final approved CPT code language will be published by the AMA in August 2019.
May 17, 2018 was a good day for the nuclear cardiology community, although there is still work to be done. Robust physician participation in RUC surveys is very important. Advocacy for our field and our patients requires constant effort.

\section{Disclosure}

Both report no conflicts of interest.

\section{References}

1. CPT Editorial Panel Summary of Actions; 2018. https://www.ama-a ssn.org/practice-management/summary-panel-actions.

2. Hirsch JA, Leslie-Mazwi TM, Nicola GN, Barr RM, Bello JA, Donovan WD, Tu R, Alson MD, Manchikanti L. Current procedural terminology: A primer. J Neurointerv Surg. 2015;7(4):309-12. https://doi.org/10.1136/neurintsurg-2014-011156.

3. Wiener DH. CPT, CMS, RUC, RBRVS: Why this alphabet soupand filling out surveys-matters to you and your practice! J Am Soc Echocardiogr. 2012;25(2):28A. https://doi.org/10.1016/j.echo.2011. 12.007. 